\title{
MEASUREMENTS OF THE MESON-PHOTON TRANSITION FORM FACTORS OF LIGHT PSEUDOSCALAR MESONS AT LARGE MOMENTUM TRANSFER
}

\author{
V. Savinov (representing the CLEO Collaboration) \\ (Contribution to the conference Photon97, Egmond aan Zee (1997)) \\ Stanford Linear Accelerator Center, \\ MS61, P.O. Box 4349, Stanford, CA, 94309, USA \\ (e-mail: savinov@lns62.lns.cornell.edu)
}

\begin{abstract}
Using the CLEO II detector, we have measured the form factors associated with the electromagnetic transitions $\gamma^{*} \gamma \rightarrow$ meson. We have measured these form factors in the momentum transfer ranges from 1.5 to 9,20 , and $30 \mathrm{GeV}^{2}$ for $\pi^{0}, \eta$, and $\eta^{\prime}$, respectively.
\end{abstract}

\section{Introduction}

Production of even $C$-parity hadronic matter in $e^{+} e^{-}$scattering provides a unique opportunity to study the properties of strong interactions. To leading order in quantum electrodynamics (QED) these processes are described as the interaction between two photons emitted by the scattered electrons. Although in $e^{+} e^{-}$scattering the probe and the target are both represented by photons that are carriers of the electromagnetic force, these space-like photons can produce a pair of quarks that interact strongly and are observed in the form of hadrons. Therefore, by measuring the four-momenta of the scattered electrons we can study the dynamics of strong interactions. The quantities of interest in these studies are the form factors associated with the transitions between the photons and the hadrons.

In this paper we briefly describe the final results of our measurements 2.2 of the differential cross sections for the production of a single pseudoscalar meson in $e^{+} e^{-}$scattering: $e^{+} e^{-} \rightarrow e^{+} e^{-\mathcal{R}}$, where $\mathcal{R}$ is a $\pi^{0}, \eta$ or $\eta^{\prime}$. We measure these cross sections in a "single-tagged" experimental mode where one of the scattered electrons is detected ("tagged"), while the other electron is scattered at a very small angle and therefore remains undetected ("untagged"). The mesons produced in $e^{+} e^{-}$scattering are observed through their decays to various fully reconstructed final states. The tagged electron emits a highly off-shell photon $\left(\gamma^{*}\right)$, whereas the untagged electron emits a nearly on-shell photon $(\gamma)$. We measure the dependence of the meson production rate on the squared momentum transfer $Q^{2}$ carried by the highly off-shell photon. This momentum transfer is determined by energymomentum conservation as applied to the tag: $Q^{2} \equiv-\left(p_{b}-p_{t}\right)^{2}=2 E_{b} E_{t}\left(1-\cos \theta_{t}\right)$, where $p_{b}$ and $p_{t}$ are the four-momenta of the incident beam-energy electron and the tag, $E_{b}$ and $E_{t}$ are corresponding energies, and $\theta_{t}$ is the scattering angle. From the measurements of the differential rates $d \sigma\left(e^{+} e^{-} \rightarrow e^{+} e^{-} \mathcal{R}\right) / d Q^{2}$ we obtain the 
transition form factors $\mathcal{F}_{\gamma^{*} \gamma \mathcal{R}}$ that describe the effect of the strong interaction in the $\gamma^{*} \gamma \rightarrow \mathcal{R}$ transition amplitudes.

To relate the differential cross sections to the transition form factors we employ the theoretical framework developed by V.M. Budnev et al. 3 (BGMS formalism). In BGMS the process $e^{+} e^{-} \rightarrow e^{+} e^{-} \mathcal{R}$ is divided into two parts: $e^{+} e^{-} \rightarrow e^{+} e^{-} \gamma^{*} \gamma$ and $\gamma^{*} \gamma \rightarrow \mathcal{R}$. The first part is completely calculable in QED and the second part is defined in terms of the transition form factors $\mathcal{F}_{\gamma^{*} \gamma \mathcal{R}}\left(Q^{2}\right)$. In the case of pseudoscalar mesons there is only one form factor. At zero momentum transfer this form factor is expressed as:

$$
\left|\mathcal{F}_{\gamma^{*} \gamma \mathcal{R}}(0)\right|^{2}=\frac{1}{(4 \pi \alpha)^{2}} \frac{64 \pi \Gamma(\mathcal{R} \rightarrow \gamma \gamma)}{M_{\mathcal{R}}^{3}},
$$

where $\alpha$ is the QED coupling constant, $M_{\mathcal{R}}$ is the mass and $\Gamma(\mathcal{R} \rightarrow \gamma \gamma)$ is the two-photon partial width of the meson $\mathcal{R}$. The transition form factors cannot be calculated directly from Quantum Chromodynamics (QCD). However, these form factors have been estimated 4.6 using theoretical methods based on the perturbative QCD (PQCD) 3 and the sum rules $\mathrm{Q}$.

Brodsky and Lepage employed PQCD to find the-asymptotic behavior of the $\gamma^{*} \gamma \rightarrow \mathcal{R}$ transition form factors in the limit $Q^{2} \rightarrow \infty 10$ :

$$
\lim _{Q^{2} \rightarrow \infty} Q^{2} \mathcal{F}_{\gamma^{*} \gamma \mathcal{R}}\left(Q^{2}\right)=2 f_{\mathcal{R}}
$$

where $f_{\mathcal{R}}$ is the meson decay constant. In addition, it has been predicted that in this limit anymessonic wave function evolves to the asymptotic wave function of unique shape 11.12 .

While PQCD predicts the form factors of the $\gamma^{*} \gamma \rightarrow \mathcal{R}$ transitions at large momentum transfer, the behavior of these form factors in the limit $Q^{2} \rightarrow 0$ can be determined from the axial anomaly 13,44 in the chiral limit of QCD. For $\pi^{0}$ and $\eta$ the axial anomaly yields 10 :

$$
\lim _{Q^{2} \rightarrow 0} \mathcal{F}_{\gamma^{*} \gamma \mathcal{R}}\left(Q^{2}\right)=\frac{1}{4 \pi^{2} f_{\mathcal{R}}}
$$

to leading order in $m_{u}^{2} / M_{\mathcal{R}}^{2}$ and $m_{d}^{2} / M_{\mathcal{R}}^{2}$ where $m_{u}$ and $m_{d}$ are the masses of the $u$ and $d$ quarks. This prediction does not hold with the same precision for $\eta^{\prime}$ due to the larger value of the $s$-quark mass. In addition, even if the $s$-quark mass were small, this prediction might be broken for $\eta^{\prime}$ because this particle is an unlikely candidate for the Goldstone boson 15.16 .

Finally, to approximate the soft non-perturbative region of $Q^{2}$ a simple interpolation between $Q^{2} \rightarrow 0$ and $Q^{2} \rightarrow \infty$ limits has been proposed 10 :

$$
\mathcal{F}_{\gamma^{*} \gamma \mathcal{R}}\left(Q^{2}\right) \sim \frac{1}{4 \pi^{2} f_{\mathcal{R}}} \frac{1}{1+\left(Q^{2} / 8 \pi^{2} f_{\mathcal{R}}^{2}\right)}
$$


We have measured the transition form factors $\mathcal{F}_{\gamma^{*} \gamma \mathcal{R}}$ in the space-like regions of the momentum transfer between 1.5 and $9 \mathrm{GeV}^{2}$ for $\pi^{0}, 1.5$ and $20 \mathrm{GeV}^{2}$ for $\eta$, and 1.5 and $30 \mathrm{GeV}^{2}$ for $\eta^{\prime}$. We report the measurements of the transition form factors of $\pi^{0}, \eta$, and $\eta^{\prime}$ using the decays: $\pi^{0} \rightarrow \gamma \gamma, \eta \rightarrow \gamma \gamma, \eta \rightarrow \pi^{0} \pi^{0} \pi^{0} \rightarrow$ $6 \gamma, \eta \rightarrow \pi^{+} \pi^{-} \pi^{0} \rightarrow \pi^{+} \pi^{-} 2 \gamma, \eta^{\prime} \rightarrow \rho^{0} \gamma \rightarrow \pi^{+} \pi^{-} \gamma, \eta^{\prime} \rightarrow \pi^{+} \pi^{-} \eta \rightarrow \pi^{+} \pi^{-} 2 \gamma$, $\eta^{\prime} \rightarrow \pi^{0} \pi^{0} \eta \rightarrow 6 \gamma, \eta^{\prime} \rightarrow \pi^{+} \pi^{-} \eta \rightarrow 2 \pi^{+} 2 \pi^{-} 2 \gamma, \eta^{\prime} \rightarrow \pi^{0} \pi^{0} \eta \rightarrow 5 \pi^{0} \rightarrow 10 \gamma$, $\eta^{\prime} \rightarrow \pi^{0} \pi^{0} \eta \rightarrow 3 \pi^{0} \pi^{+} \pi^{-} \rightarrow \pi^{+} \pi^{-} 6 \gamma$, and $\eta^{\prime} \rightarrow \pi^{+} \pi^{-} \eta \rightarrow \pi^{+} \pi^{-} 3 \pi^{0} \rightarrow \pi^{+} \pi^{-} 6 \gamma$. We have analyzed the last two decay chains of $\eta^{\prime}$ together since they are observed in the same final state $\pi^{+} \pi^{-} 6 \gamma$.

The data sample employed in our analysis corresponds to an integrated $e^{+} e^{-}$ luminosity of $2.88 \pm 0.03 \mathrm{fb}^{-1}$ collected at $e^{+} e^{-}$center-of-mass energy around 10.6 $\mathrm{GeV}$ with the CLEO-II detector 17.18 at CESR.

\section{Analysis Procedure}

To measure the products of the differential cross sections and branching fractions for each decay chain we puse the following analysis procedure. Data events that pass all selection criterial 3 are used to form the $Q^{2}$ distribution where the value of $Q^{2}$ for each event is estimated from energy-momentum conservation for this event (the experimental method we use to estimate the value of $Q^{2}$ for each event is described in detail elsewhere 1,2). Next we divide the event yields into $Q^{2}$ intervals. For each $Q^{2}$ interval we obtain the number of signal events in data from the fit to the invariant mass distribution. Then we estimate and subtract the (small) feed-down background 2 . Finally we correct the background-subtracted number of signal events for the detection efficiency. The signal line shapes used in the fits and the detection efficiencies are determined from the detector simulation for each $Q^{2}$ interval. To extract the transition form factors we compare the measured and the predicted values of the cross sections. Namely, for each $Q^{2}$ interval, we measure the form factors $\mathcal{F}_{\gamma^{*} \gamma \mathcal{R}}^{\text {data }}\left(\tilde{Q}^{2}\right)$ from: $\left|\mathcal{F}_{\gamma^{*} \gamma \mathcal{R}}^{\text {data }}\left(\tilde{Q}^{2}\right)\right|^{2}=\sigma($ data $) / \sigma(M C)\left|\mathcal{F}_{\gamma^{*} \gamma \mathcal{R}}^{M C}\left(\tilde{Q}^{2}\right)\right|^{2}$, where $\mathcal{F}_{\gamma^{*} \gamma \mathcal{R}}^{M C}\left(\tilde{Q}^{2}\right)$ is the approximation for the $Q^{2}$-dependent part of the form factor in Monte Carlo (MC) simulation, and $\sigma($ data) and $\sigma(M C)$ are the cross sections for this $Q^{2}$ interval measured in data and predicted using numerical integration, respectively. The transition form factors are measured at $\tilde{Q}^{2}$ where the differential cross sections achieve their mean values according to the results of numerical integration.

The $Q^{2}$-dependent part of the $\gamma^{*} \gamma^{*} \rightarrow \mathcal{R}$ transition form factors in our twophoton $\mathrm{MC}$ simulation program is approximated by:

$$
\left|\mathcal{F}_{\gamma^{*} \gamma^{*} \mathcal{R}}\left(Q^{2}\right)\right|^{2}=\frac{1}{(4 \pi \alpha)^{2}} \frac{64 \pi \Gamma(\mathcal{R} \rightarrow \gamma \gamma)}{M_{\mathcal{R}}^{3}} \frac{1}{\left(1+Q^{2} / \Lambda_{\mathcal{R}}^{2}\right)^{2}}
$$

where the pole-mass parameter $\Lambda_{\mathcal{R}}=770 \mathrm{MeV}$. 


\section{Results}

In Figures 1 and 2 we compare our results with the theoreticalpredictions. In these figures we also show the results of the CELLO experiment 19 and the asymptotic prediction of PQCD given by Eqn. 2. For both experimental results the error bars represent the statistical errors only. To plot the results of the theoretical predictions we use their published analytical forms. To estimate the values of the meson decay constants $f_{\mathcal{R}}$ we use Eqns. 11 and 3 and the tabulated two-photon partial widths of the studied mesons 20 .

Finally, for each meson $\mathcal{R}$, where $\mathcal{R}$ is $\pi^{0}, \eta$, or $\eta^{\prime}$, we derive the values of the pole-mass parameters $\Lambda_{\mathcal{R}}$ which we use to represent our results in a simple phenomenological form. For each meson we fit all our results for $\left|\mathcal{F}_{\gamma^{*} \gamma \mathcal{R}}\left(Q^{2}\right)\right|^{2}$ with the function given by Eqn. 5 and obtain the values of the pole-mass parameters $\Lambda_{\mathcal{R}}$ shown in Table 1. In this table, for each measurement, the first error is statistical, the second error represents the systematic uncertainties of our measurement and the third error reflects the experimental error in the value of the two-photon partial width of the meson. The results of the fits are also shown in Figures 11 and 2 .

We use the measured values of the parameters $\Lambda_{\pi^{0}}$ and $\Lambda_{\eta}$ to compare the soft non-perturbative properties of $\pi^{0}$ and $\eta$. This is a legitimate comparison because the asymptotic prediction given by Eqn. 2 and the chiral limit given by Eqn. 3 are expected to hold for both $\pi^{0}$ and $\eta$. From the comparison between the measured values of $\Lambda_{\pi^{0}}$ and $\Lambda_{\eta}$ we conclude that the $Q^{2}$ shapes of the $\gamma^{*} \gamma \rightarrow$ meson transition form factors of $\pi^{0}$ and $\eta$ are nearly identical, which strongly indicates the similarity between the wave functions of these mesons.

The results of our measurements for the production of $\eta^{\prime}$ demonstrate that if this particle were a $q \bar{q}$ bound state and the QCD chiral limit given by Eqn. 3 held for this meson, the $Q^{2}$-dependence of the transition form factor of $\eta^{\prime}$ and consequently its wave function would be significantly different from these non-perturbative properties of either $\pi^{0}$ or $\eta$.

\section{Conclusions}

We have measured the form factors associated with the electromagnetic transitions $\gamma^{*} \gamma \rightarrow$ meson in the regions of momentum transfer from 1.5 to 9,20 , and $30 \mathrm{GeV}^{2}$ for $\pi^{0}, \eta$, and $\eta^{\prime}$, respectively. These are the first measurements above $2.7 \mathrm{GeV}^{2}$ for $\pi^{0}$ and above $7 \mathrm{GeV}^{2}$ for $\eta$ and $\eta^{\prime}$.

Our measurement for $\pi^{0}$ unambiguously distinguishes among various theoretical predictions for the form factors of the $\gamma^{*} \gamma \rightarrow \pi^{0}$ transition. We have demonstrated that the non-perturbative properties of $\pi^{0}$ and $\eta$ agree with each other which indicates that the wave functions of these two mesons are similar. In the $\eta^{\prime}$ analysis we have shown that the non-perturbative properties of $\eta^{\prime}$ differ substantially from those of $\pi^{0}$ and $\eta$. Our measurement for $\eta^{\prime}$ provides important information for 
future theoretical investigations of the structure of this particle.

\section{References}

1. V. Savinov, PhD thesis, University of Minnesota, Minneapolis, 1997, Unpublished.

2. J. Gronberg et al., CLEO Coll., CLNS 97/1477, Submitted to PRD.

3. V.M. Budnev et al., Phys. Rep. C15 (1975) 181.

4. P. Kroll, Universität Wuppertal preprint WU B 94-17..

5. R. Jakob et al., J. Phys. G22 (1996) 45.

6. F.-G. Cao et al., Phys. Rev. D53 (1996) 6582.

7. A.V. Radyushkin and R. Ruskov, Nucl. Phys. B481 (1996) 625.

8. G.P. Lepage and S.J. Brodsky, Phys. Rev. D22 (1980) 2157.

9. M.A. Shifman, et al., Nucl. Phys. B147 (1979) 385.

10. S.J. Brodsky and G.P. Lepage, Phys. Rev. D24 (1981) 1808.

11. A.V. Efremov and A.V. Radyushkin, Phys. Lett. B94 (1980) 245.

12. V.L. Chernyak and A.R. Zhitnitsky, Phys. Rep. 112 (1984) 173.

13. S. Adler, Phys. Rev. 177 (1969) 2426.

14. J. Bell and R. Jackiw, Nuovo. Cim. A60 (1969) 47.

15. L.H. Ryder, Quantum Field Theory, p.297, Cambridge University Press, 1986.

16. S. Coleman, Aspects of Symmetry, Cambridge University Press, 1985.

17. Y. Kubota et al., CLEO Coll., Nucl. Inst. and Meth. A320 (1992) 66.

18. C. Bebek et al., CLEO Coll., Nucl. Inst. and Meth. A302 (1992) 261.

19. H.-J. Behrend et al., CELLO Coll., Z. Phys. C49 (1991) 401.

20. Particle Data Group, L. Montanet et al., Review of Particle Properties, Phys. Rev. D54 (1996) 1.

Table 1: Values of the pole-mass parameters $\Lambda_{\pi^{0}}, \Lambda_{\eta}$ and $\Lambda_{\eta^{\prime}}$.

\begin{tabular}{cc}
\hline Decay chain & $\Lambda_{\mathcal{R}}(\mathrm{MeV})$ \\
\hline$\pi^{0} \rightarrow \gamma \gamma$ & $776 \pm 10 \pm 12 \pm 16$ \\
\hline Simultaneous fit to three decay chains for $\eta$ & $774 \pm 11 \pm 16 \pm 22$ \\
\hline Simultaneous fit to seven decay chains for $\eta^{\prime}$ & $859 \pm 9 \pm 18 \pm 20$ \\
\hline
\end{tabular}



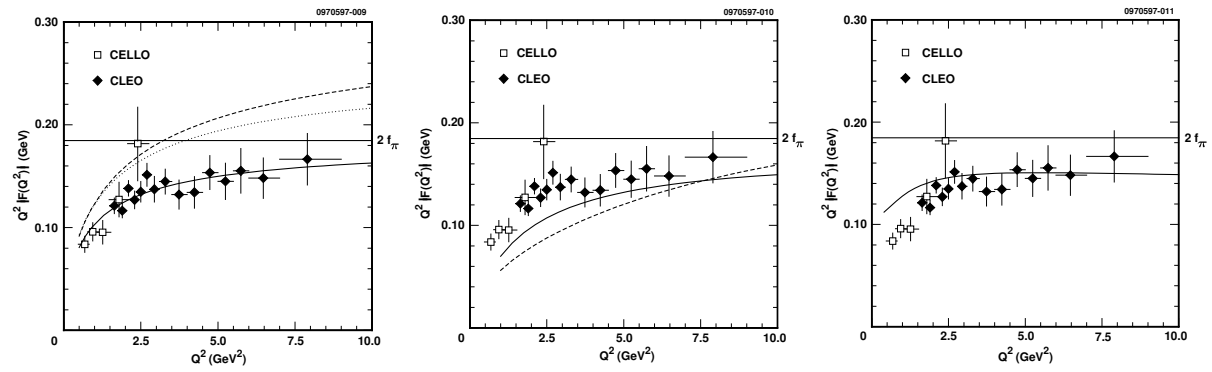

Figure 1: Comparison of the results (points) for $\pi^{0}$ with the theoretical predictions 6 , (fropt to right). Two figures on the left show the predictions with the asymptotic wave function 11.12 (solid curves) and the $\mathrm{CZ}$ wave function 12 (dashed curves). The dotted curve shows the prediction made with the $\mathrm{CZ}$ wave function when its QCD evolution is taken into account.
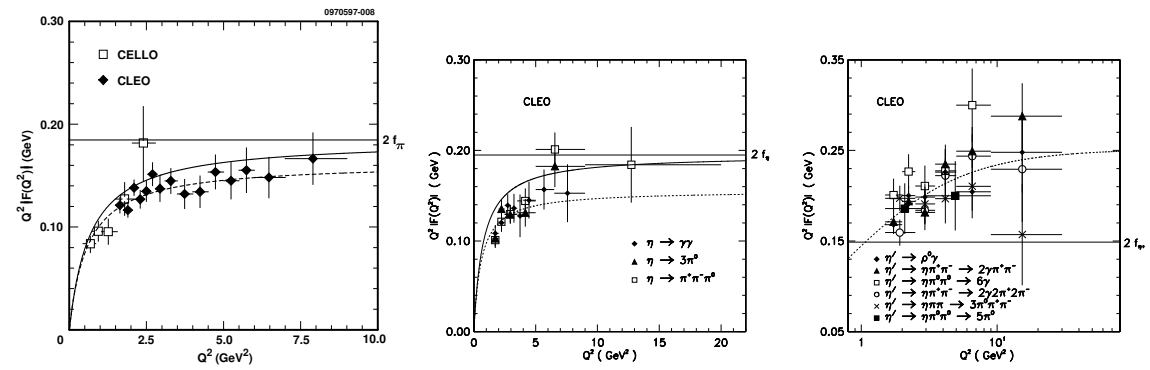

Figure 2: Pole-mass parameter fits (dashed lines) to CLEO results for (from left to right) $\pi^{0}, \eta$, and $\eta^{\prime}$. The solid curves (where shown) are the interpolations given by Eqn. 